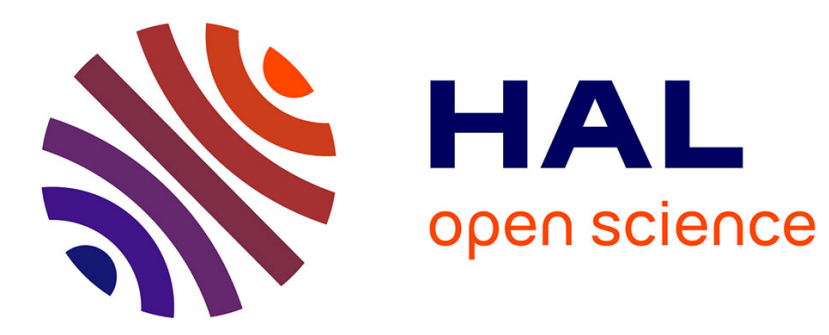

\title{
Contrast enhancement in polarimetric imaging with correlated noise fluctuations
}

\author{
Swapnesh Panigrahi, Julien Fade, Mehdi Alouini
}

\section{To cite this version:}

Swapnesh Panigrahi, Julien Fade, Mehdi Alouini. Contrast enhancement in polarimetric imaging with correlated noise fluctuations. Max ENT, Sep 2014, Amboise, France. pp.25. hal-01065627

\section{HAL Id: hal-01065627}

\section{https://hal-univ-rennes1.archives-ouvertes.fr/hal-01065627}

Submitted on 17 Dec 2014

HAL is a multi-disciplinary open access archive for the deposit and dissemination of scientific research documents, whether they are published or not. The documents may come from teaching and research institutions in France or abroad, or from public or private research centers.
L'archive ouverte pluridisciplinaire HAL, est destinée au dépôt et à la diffusion de documents scientifiques de niveau recherche, publiés ou non, émanant des établissements d'enseignement et de recherche français ou étrangers, des laboratoires publics ou privés. 


\title{
Contrast enhancement in polarimetric imaging with correlated noise fluctuations
}

\author{
Swapnesh Panigrahi, Julien Fade and Mehdi Alouini \\ Institut de Physique de Rennes, CNRS, Université de Rennes 1, Campus de Beaulieu, 35042 \\ Rennes, France
}

\begin{abstract}
We compare the measurement precision of a polarimetric camera to that of a simple intensity camera when imaging a partially polarized light-mark embedded in an intense and partially polarized background. We show that the gain in measurement precision while using a polarimetric camera is maximized when the noise fluctuations on the two polarimetric channels are significantly correlated. Further, we implement a snapshot polarimetric camera for long distance imaging of a highly polarized light source through fog and compare the contrast obtained using various representations of the polarimetric images. We show that the representation that provides the best contrast depends on the visibility conditions and matches well with theoretical predictions.
\end{abstract}

Keywords: Polarimetric imaging, Fisher information, Noise in imaging systems PACS: 42.30.Va; 42.30.Tz; 89.70.Cf

\section{INTRODUCTION}

In polarimetric sensitive imaging, the polarimetric properties of light emitted, reflected or transmitted by objects in a scene are recorded. The polarimetric data is further processed to enhance the contrast of non-uniformities in polarization parameters such as degree of polarization (DOP), retardance or diattenuation magnitude and angle etc. Generally, in polarimetric imaging, a scene is illuminated using a light source and the reflected light is recorded using a polarization sensitive detector (PSD). The reflection data can provide information about the surface properties of the object being imaged. The polarization properties of light reflected from a surface depends on its granularity and therefore can help in distinguishing between materials with different surface properties [1]. Polarization sensitive imaging has been used in various fields that include medical diagnostics [3], industrial quality control [1, 4], machine vision [5], remote sensing [6] and imaging through turbid medium (e.g. fog, turbid and colloidal solutions) $[7,8]$. In this work, we consider a polarized source of light which is used as 'signal' and we aim at enhancing the visibility of this source to efficiently isolate it from the surrounding scene. This has tremendous application in navigation [2]. Generally, in the applications mentioned above, polarimetric imaging brings more information about the scene being imaged so that the contrast of sub-regions in a given scene can be enhanced. In the course of this article we quantify the gain in measurement precision that can be obtained using a PSD with respect to a simple intensity detector (ID). We also implement an active polarimetric imaging system to enhance the contrast of a polarized light-mark through atmospheric fog from a distance of about $1.3 \mathrm{~km}$. Such long distance imaging can be helpful in air and sea navigation for providing visual aid during low visibility conditions. 


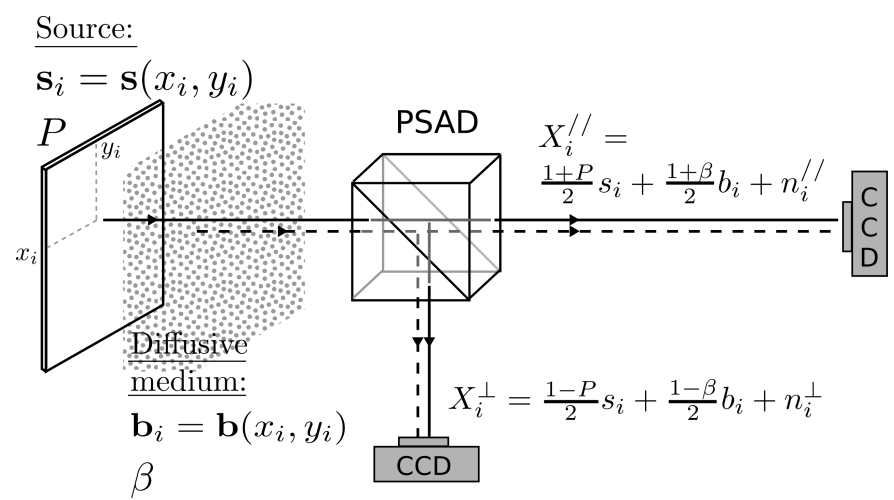

FIGURE 1. A generic schematic of the image formation model. A polarization-splitting analyzing device (PSAD) produces two simultaneous images of a partially polarized light source through a turbid medium [12].

\section{POLARIMETRIC CONTRAST IMAGING}

To achieve polarization sensitive imaging, the four-dimensional Stokes' vector $\left(\left[S_{0}, S_{1}, S_{2}, S_{3}\right]^{T}\right)$ of the incoming light must be measured at each pixel. The Stokes' vector is obtained as given below

$$
\mathbf{S}=\left(\begin{array}{c}
S_{0} \\
S_{1} \\
S_{2} \\
S_{3}
\end{array}\right)=\left(\begin{array}{c}
\left\langle E_{x}^{2}\right\rangle+\left\langle E_{y}^{2}\right\rangle \\
\left\langle E_{x}^{2}\right\rangle-\left\langle E_{y}^{2}\right\rangle \\
\left\langle E_{+45^{\circ}}^{2}\right\rangle-\left\langle E_{-45^{\circ}}^{2}\right\rangle \\
\left\langle E_{R}^{2}\right\rangle-\left\langle E_{L}^{2}\right\rangle
\end{array}\right)
$$

where the indices $x$ and $y$ represent two orthogonal Cartesian axes and $R$ and $L$ represent right and left circularly polarized light. The degree of polarization (DOP) of the source is obtained by using the relation, $D O P=\sqrt{S_{1}^{2}+S_{2}^{2}+S_{3}^{2}} / S_{0}$. However, in the case where the intervening medium is non-birefringent, only two components of the Stokes' vector are enough to estimate the DOP of light at each pixel by calculating the so-called orthogonal states contrast (OSC) given by

$$
O S C=\frac{S_{1}}{S_{0}}=\frac{X^{\|}-X^{\perp}}{X^{\|}+X^{\perp}},
$$

where $X_{\|}$and $X_{\perp}$ are images of the same scene taken in two orthogonal polarization directions. Using such a polarimetric imaging scheme, it is possible to implement various image representations to enhance the contrast of polarimetric non-uniformities in a given scene. Some of the widely used representations that work with varying degrees of performance are OSC image (denoted by $\gamma_{O S C}=\left(X^{\|}-X^{\perp}\right) /\left(X^{\|}+X^{\perp}\right)$ ) [10] and polarization difference image (denoted by $\gamma_{\Delta}=X_{\|}-X_{\perp}$ ) [9]. In general, it is interesting to quantitatively compare the best contrast that can be obtained using polarimetric imaging w.r.t. contrast obtained using a simple intensity imager. Consequently, a comparison of the various image representations can be helpful in deciding which representation should be used for real-time imaging situations. 


\section{IMAGING SCHEME AND NOISE MODEL}

The problem that we address here consists of imaging an incoherent source of partially polarized light through a non-birefringent medium using polarization sensitive imaging as shown in the schematic in Fig. 1. At the $i^{\text {th }}$ pixel of the image retrieved in this generic imaging scheme, we consider a light source of intensity $s_{i}$ and DOP $P \in[0,1]$ embedded in an intense background with intensity $b_{i}$ and DOP $\beta$. A polarizationsplitting analyzing device (PSAD) creates two images of the same scene in orthogonal polarization directions forming a polarimetric image $X^{P}=\left[X^{\|}, X^{\|}\right]^{T}$ consisting of a set of two-dimensional pixels with the $i^{t h}$ pixel given by $X_{i}^{P}=\left[x_{i}^{\|}, x_{i}^{\perp}\right]^{T}$. A part of the noise in each channel can be attributed to the detector noise (with noise variance denoted by $\sigma_{0}^{2}$ ) which remains uncorrelated in the two channels. Further noise contribution arises from the optical fluctuations that are a result of the turbulence and scattering properties or spatial/temporal inhomogeneities of the intervening medium. The noise variance introduced by the 'optical noise' is denoted by $\varepsilon_{i}^{2}$. Since the 'optical noise' arises due to background optical intensity fluctuation, the noise contribution at each channel depends on the background DOP $\beta$ and the average background intensity $b$. Thus, the scenedependent fluctuations in the two image channels are likely to be correlated. In light of this, we consider a bi-dimensional Gaussian noise model for each pixel, with mean intensity $\left\langle X_{i}^{P}\right\rangle=\left[\frac{1+P}{2} s_{i}+\frac{1+\beta}{2} b_{i}, \frac{1-P}{2} s_{i}+\frac{1-\beta}{2} b_{i}\right]^{T}$ and whose second order statistical properties are given by the covariance matrix

$$
\Gamma_{i}=\left(\begin{array}{cc}
\frac{1+\beta}{2} \varepsilon_{i}^{2}+\sigma_{0}^{2} & \rho \frac{\sqrt{1-\beta^{2}}}{2} \varepsilon_{i}^{2} \\
\rho \frac{\sqrt{1-\beta^{2}}}{2} \varepsilon_{i}^{2} & \frac{1-\beta}{2} \varepsilon_{i}^{2}+\sigma_{0}^{2}
\end{array}\right),
$$

with $\rho$ denoting a correlation parameter.

We assume a Gaussian probability density function for the $\mathrm{N}$-pixels measurement sample which is given by $P\left(X^{P}\right)=\Pi_{i=1}^{N} \exp \left\{-\frac{1}{2}\left(\delta X_{i}^{P}\right)^{T} \Gamma^{-1} \delta X_{i}^{P}\right\} / 2 \pi \sqrt{\operatorname{det}\left(\Gamma_{i}\right)}$ where $\delta X_{i}^{P}=X_{i}^{P}-\left\langle X_{i}^{P}\right\rangle$. Similarly, if the PSD is replaced with a simple intensity detector (ID), the mean pixel intensity is given by $\left\langle X_{i}^{I}\right\rangle=s_{i}+b_{i}$ with a variance of $\sigma_{0}^{2}+\varepsilon_{i}^{2}$. Using the above definitions and assumptions, we consider a general framework consisting of estimating the parameter ' $s$ ' with minimum variance. The maximum achievable gain in precision in each imaging modality can then be compared fairly. Consequently, we determine the Fisher information (FI) with respect to the parameter ' $s$ ' for each imaging modality and define their ratio (FI in polarimetric imaging to that of intensity imaging) as the gain.

\section{GAIN IN MEASUREMENT PRECISION}

The Fisher information, defined in Eq.(4) is a measure of the amount of information available in sample $\mathbf{X}$ for estimation of a parameter $y$ and its inverse gives the Cramer- 
Rao bound (CRB) which is the lower bound on the variance in estimating $y$.

$$
I_{F}(y)=-\left\langle\frac{\partial^{2} \ln P_{\mathbf{X}}(\mathbf{X})}{\partial y^{2}}\right\rangle
$$

Thus, the ratio between the Fisher information calculated for a PSD and an ID gives the maximum gain achievable by use of a polarimetric camera. A detailed derivation of the FI for each imaging modality is reported in [12] and the simplified expression of the gain is presented here as $\mu$

$$
\mu=\frac{I_{F}^{P}(s)}{I_{F}^{I}(s)}=\frac{\left(1+\omega^{2}\right)\left[\frac{1+P^{2}}{2}+\frac{Q}{4} \omega^{2}\right]}{1+\omega^{2}+\frac{\left(1-\rho^{2}\right)\left(1-\beta^{2}\right)}{4} \omega^{4}},
$$

where,

$$
Q=1-2 \beta P+P^{2}-\rho\left(1-P^{2}\right) \sqrt{1-\beta^{2}}
$$

and the variable $\omega^{2}=\varepsilon^{2} / \sigma_{0}^{2}$ is introduced in the above expressions to represent the ratio of 'optical noise' to detector noise.

\section{ANALYSIS OF GAIN $\mu(\omega, P, \beta, \rho)$}

Firstly, a tedious but feasible calculation shows that the gain is a monotonically increasing function of $\omega$. This indicates that it is indeed favorable to use polarimetric imaging when the case considered has intense background levels as $\omega$ is assumed to be dependent on $b$. However when detector noise dominates (i.e. $\omega<<1$ ), the gain falls below unity, since $\mu(\omega<<1, P, \beta, \rho) \rightarrow\left(1+P^{2}\right) / 2 \leq 1$. Then, we consider the case there the detector noise is negligible as compared to the background levels and 'optical noise' i.e. where $\omega \rightarrow \infty$. In this asymptotic case, the gain expression simplifies to

$$
\mu_{\infty}(P, \beta, \rho)=\mu(\omega \rightarrow \infty P, \beta, \rho)=\frac{Q}{\left(1-\rho^{2}\right)\left(1-\beta^{2}\right)},
$$

In real-time situations the correlation between the two polarimetric channels may vary between 0 and 1 depending on turbulence and density of the scattering medium. In the presence of natural or man-made objects in the background, the visibility of the objects through the scattering medium will also give rise to variations in the correlation parameter $\rho$. Thus, we map out the various values of $P$ and $\beta$ for which the gain reaches a value $K$ (with $K \geq 1$ ) at any value of $\rho$. The following conditions present the constraints on $P$ and $\beta$ :

$$
\begin{aligned}
& \beta \leq \frac{(1+P)^{2}}{2 K}-1, \text { if } \beta \leq P \\
& \beta \geq 1-\frac{(1-P)^{2}}{2 K}, \text { if } \beta \geq P .
\end{aligned}
$$

These conditions are presented in form of a contour chart in Fig. 2. The shaded regions in the figure show conditions favorable for polarimetric imaging. The figure indicates 

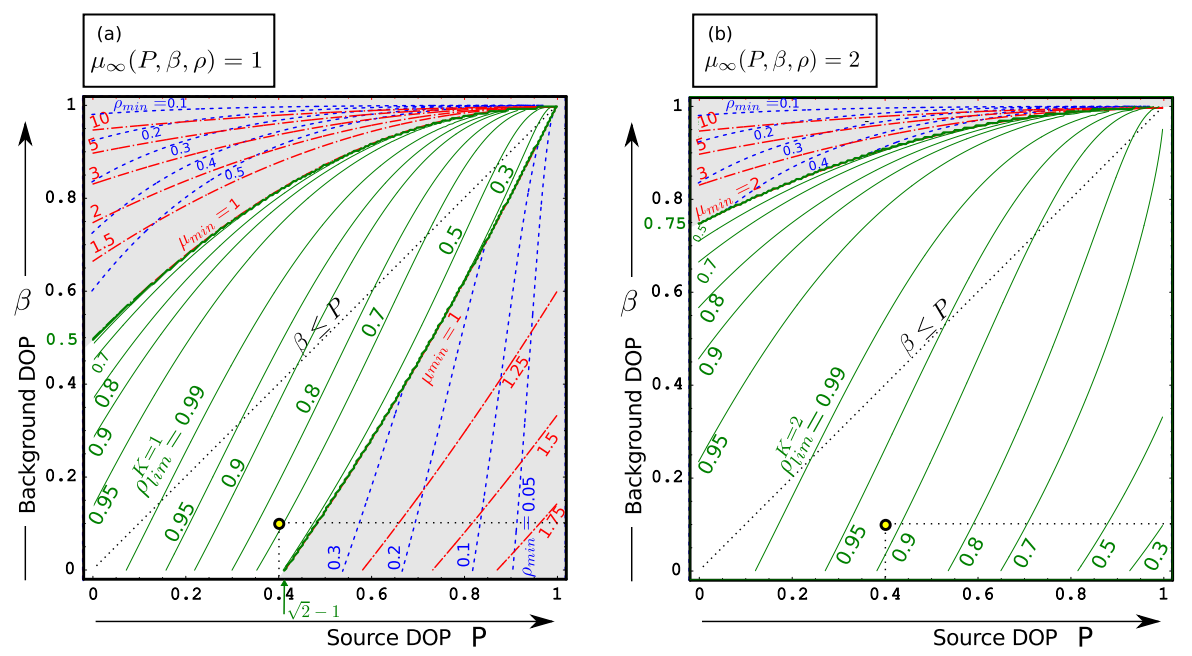

FIGURE 2. The contour plots show the range of minimal values of $\rho$ as a function of $P$ and $\beta$ for which a specified gain can be achieved, in case of negligible detector noise [12].

that the gain is greater than unity when the DOP contrast between the source and the background are high.

\section{OPTIMAL ESTIMATOR}

The above gain calculations present a maximum achievable gain that corresponds to an optimal representation of image in each modality. In light of this, we also derive estimators of $s$ in the maximum likelihood (ML) sense, since ML estimators are known to be efficient under Gaussian fluctuations. Limiting ourselves to the asymptotic case of $\omega \rightarrow \infty$, we present here the expression for the optimal estimator which is thoroughly derived in [12]. The expression of the estimator takes a form of a simple linear representation given by

$$
\hat{s}_{M L}^{P}=\frac{U \hat{X}^{\|}+V \hat{X}^{\perp}+Z}{W}
$$

where $U, V, W$ and $Z$ are functions of $P, \beta, \rho$ and $b$ [12], which are assumed $a$ priori known. Further, it can be shown that the ML estimator $\hat{s}_{M L}^{P}$ is equivalent to a difference estimator, $\hat{s}_{\Delta}^{P}$, only when $\rho=(1-\beta P) /\left(1-\beta^{2}\right)$.

\section{A SNAPSHOT POLARIMETRIC CAMERA}

We implement a Wollaston prism-based snapshot polarimetric camera [11, 13] to allow for real-time polarimetric imaging. The schematic of the camera and its image are shown in Fig. 3[a] and [b], respectively. The Wollaston prism (WP) splits the incoming light into orthogonal polarizations with a split angle of $5^{\circ}$. The resulting two images, namely $I_{\|}$and $I_{\perp}$ are recorded over a CCD camera and are extracted using the image calibration method described in [13]. A pixel-to-pixel image registration between the two images 

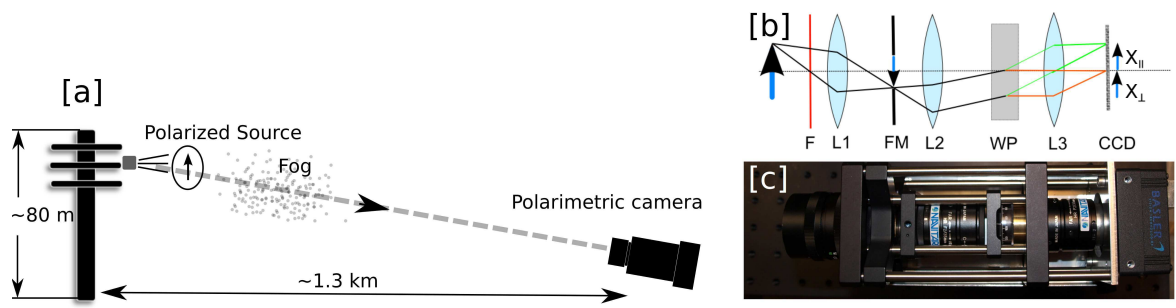

FIGURE 3. [a] The schematic shows the long distance polarimetric imaging setup through fog. The principle of the Wollaston-based snapshot polarimetric camera is shown in [b] accompanied with a picture of the actual arrangement in [c].

provides us with the 2D polarimetric image. We employ such a snapshot polarimetric camera for a kilometric-range imaging experiment which is briefly described below.

\section{LONG-RANGE EXPERIMENTAL SETUP: ENHANCED VISION THROUGH FOG}

We set up an active polarimetric imaging experiment using an incoherent source of highly polarized light placed on a nearby telecommunication tower (height $\sim 80 \mathrm{~m}$ ) at a distance of about $1.3 \mathrm{~km}$ away from the polarimetric camera. An in-house LabVIEW program is employed for auto-exposure control and data is acquired during a foggy day with each frame taken at an interval of 10 seconds because of storage constraints. As a preliminary step, the contrast obtained from four simple linear representations are compared with each other. We identify four commonly used linear combinations: difference image (denoted by $\gamma_{\Delta}=X_{\|}-X_{\perp}$ ), OSC image (denoted by $\left.\gamma_{O S C}=\left(X_{\|}-X_{\perp}\right) /\left(X_{\|}+X_{\perp}\right)\right)$, intensity-summed image $\left(\gamma_{\Sigma}=X_{\|}+X_{\perp}\right)$ and polarizationfiltered image $\left(\gamma_{\|}=X_{\|} /\left\langle X_{\perp}\right\rangle\right)$ which is 'smoothed' by dividing by the mean graylevel of $X^{\perp}$. These representations have different scaling in gray levels. To overcome this and to compare the contrast of the source with respect to the background, we use contrast-tonoise ratio $(\mathrm{CNR})$ as the contrast function. We extract a $21 \times 21$ pixel region-of-interest around the source and identify a $3 \times 3$ pixels central region as the source (denoted by S) . The rest of the pixels within the $21 \times 21$ pixels region are identified as background (B). The CNR is calculated using the following expression:

$$
C N R=\frac{\langle\gamma\rangle_{\mathbf{S}}-\langle\gamma\rangle_{\mathbf{B}}}{\sqrt{\frac{1}{N_{\mathbf{B}}-1} \sum_{i \in \mathbf{B}}^{N_{\mathbf{B}}}\left(\gamma_{i}-\langle\gamma\rangle_{\mathbf{B}}\right)^{2}}},
$$

where $\langle\gamma\rangle_{\chi}$ is the average gray level over a region $\chi$ with cardinality $N_{\chi}$.

\section{Day-time measurements}

For a single dataset acquired on 17-10-2011 between 10.02 am to 10.57 am, we extract the region of interest and analyze the evolution of source contrast with the 

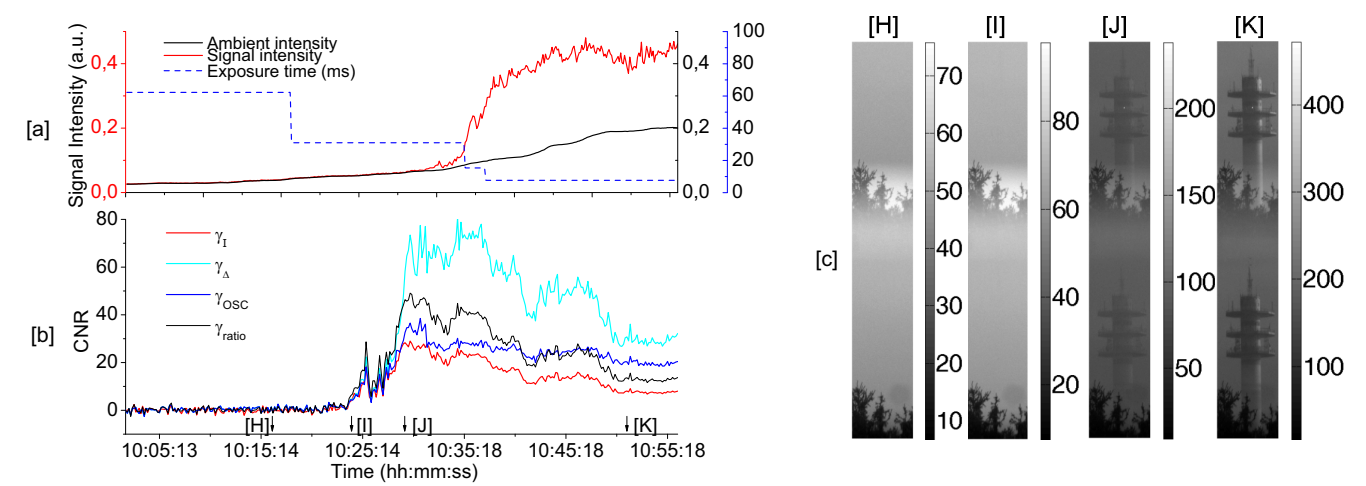

FIGURE 4. The evolution of ambient intensity, signal intensity and auto-exposure response is shown in [a] while the evolution of CNR obtained from each representation is shown in [b]. [c] shows a few raw images for the time stamps that are labeled in [b]. [13]

TABLE 1. CNR values for each representation at times labeled $\mathrm{H}$ through $\mathrm{K}$ in Fig. 4

\begin{tabular}{lrrrr}
\hline & {$[\mathbf{H}]$} & {$[\mathbf{I}]$} & {$[\mathbf{J}]$} & {$[\mathbf{K}]$} \\
\hline$\gamma_{\Sigma}$ & 0.45 & 3.33 & 27.49 & 8.71 \\
$\gamma_{\Delta}$ & 0.94 & 3.55 & 64.63 & 31.61 \\
$\gamma_{O S C}$ & -0.95 & 3.35 & 34.60 & 21.10 \\
$\gamma_{\|}$ & 0.21 & 4.96 & 45.71 & 14.61 \\
$\gamma_{M L}$ & 0.38 & 5.00 & 63.09 & 29.53 \\
\hline
\end{tabular}

change in visibility for each of the four representations enumerated in the previous section. A comparison plot is presented in Fig. 4(c). The CNR for each representation is close to zero at time $[\mathrm{H}]$ and rises with increase in visibility as the fog thins out at around 10:25 am (labeled as [I]). When the visibility increases due to thinner fog (for times between labels $[\mathrm{J}]$ and $[\mathrm{K}]$ ), the difference image $\gamma_{\Delta}$ clearly has better source contrast than other representations. The OSC image $\gamma_{O S C}$, which is commonly used in polarimetric imaging does not provide the best contrast, in fact, the polarization filtered image $\gamma_{\|}$outperforms the OSC in some case. The contrast obtained by using $\gamma_{O S C}$ has been shown to be worse during night-time measurements in [13]. The CNR values at the labeled time-stamps are given in the table 1, where it can be noticed that during low visibility conditions (time label $[\mathrm{I}]$ ), the polarization filtered image marginally outperforms all other representations. The experimental results indicate that the four representations used here do not consistently provide the best representation. However, by using the ML representation (given in Eq.(10)) suitable to the current experimental conditions $(P=1, \beta=0)$, one can consistently obtain signal contrasts close to the best achievable contrast irrespective of the visibility condition. The CNR values obtained using the ML representation (denoted by $\gamma_{M L}$ ) are also shown in Table 1 . These values are close to the best representation in each case. The small difference may be attributed 
to the fact that the the detector noise is neglected in the ML representation used and that the correlation coefficient between the two polarimetric channels are estimated over a small neighbourhood of the signal pixel. These preliminary results pave way for detailed investigation for obtaining contrast maximizing representations in real-time. The simple linear form of the representation also makes it feasible for implementation on a standalone device with real-time capabilities.

\section{CONCLUSION}

As a conclusion, the theoretical results suggest that the snapshot polarimetric imagers can significantly improve the estimation precision because the simultaneous acquisition of images can lead to correlated noise in the polarimetric channels. It is also shown, both theoretically and experimentally, that the optimal representation differs, in general, from a simple difference image or OSC image. The derived estimator can be easily implemented and paves way for further investigation, specifically, on unsupervised detection procedures for real-time assistance in navigation through obscured weather. These results can be useful for the design of polarimetric imaging systems involving estimation through turbid media, or in other fields of application, for post-processing of polarimetric images exhibiting temporally or spatially correlated fluctuations.

\section{ACKNOWLEDGMENTS}

We thank Dr. Hema Ramachandran (Raman Research Institute, Bangalore, India) and Dr. Fabien Bretenaker (Laboratoire Aimé Cotton, Orsay, France) for the valuable discussions. The authors are grateful to the TDF company for providing a location for the emitter on the telecommunication tower. This work has been partly funded by the CEFIPRA (project no. 4604-4).

\section{REFERENCES}

1. P. Terrier, V. Devlaminck, and J. Charbois, J. Opt. Soc. Am. A 25, 423-430 (2008).

2. M. Yamada, K. Ueda, I. Horiba, and N. Sugie, IEEE Trans. Intell. Transp. Syst. 2, 26-31 (2001).

3. S. Demos, H. Savage, A. S. Heerdt, S. Schantz, and R. Alfano, Opt. Commun. 124, 439-442 (1996).

4. O. Morel, C. Stolz, F. Meriaudeau, and P. Gorria, Appl. Opt. 45, 4062-4068 (2006).

5. G. Anna, N. Bertaux, F. Galland, F. Goudail, and D. Dolfi, Opt. Lett. 37, 3321-3323 (2012).

6. M. Dubreuil, P. Delrot, I. Leonard, A. Alfalou, C. Brosseau, and A. Dogariu, Appl. Opt. 52, 997-1005 (2013).

7. O. Emile, F. Bretenaker, and A. L. Floch, Opt. Lett. 21, 1706-1708 (1996).

8. H. Ramachandran and A. Narayanan, Opt. Commun. 154, 255-260 (1998)

9. M. P. Rowe, J. S. Tyo, N. Engheta, and E. N. Pugh, Opt. Lett. 20, 608-610 (1995).

10. A. Bénière, F. Goudail, M. Alouini, and D. Dolfi, J. Opt. Soc. Am. A 25, 919-929 (2008).

11. A. Bénière, M. Alouini, F. Goudail, and D. Dolfi, Appl. Opt. 48, 5764-5773 (2009).

12. J. Fade, S. Panigrahi, and M. Alouini, Opt. Express 22, 4920-4931 (2014).

13. J. Fade, S. Panigrahi, A. Carré, L. Frein, C. Hamel, F. Bretenaker, H. Ramachandran, and M. Alouini, Appl. Opt. 53, 3854-3865 (2014). 